\title{
Rubinstein-Taybi Syndrome
}

National Cancer Institute

\section{Source}

National Cancer Institute. Rubinstein-Taybi Syndrome. NCI Thesaurus. Code C75466.

A rare genetic syndrome mapped to chromosome 16p13.3 and associated with mutations in the CREBBP gene. It is characterized by mental and growth retardation, distinctive facial features (prominent nose, low-set ears, microcephaly, and small mouth), and broad thumbs and great toes. Patients are at an increased risk of developing benign and malignant neoplasms, including nervous system neoplasms and malignant lymphoproliferative disorders. 